\title{
MOTION \\ Special relativity and Kepler's first law
}

Consider a two-body system consisting of a very massive sun and a planet in a circular orbit of radius $R$ around it, as seen by an inertial observer $S$ at rest with respect to the sun. Obviously this sun-planet system obeys Kepler's first law. For concreteness let the planet revolve on the plane $z=0$ and the sun be located at $x=y=z=0$. Now consider another inertial observer $S^{\prime}$ moving with speed $\vec{v}=c \beta \hat{x}$, say, on the plane $z=z_{s}$ relative to the sun. The Lorentz transformations relating to the inertial observers mentioned are

$$
\begin{aligned}
c t & =\gamma\left(c t^{\prime}+\beta x^{\prime}\right) \\
x & =\gamma\left(x^{\prime}+\beta c t^{\prime}\right) \\
y & =\mathrm{y}^{\prime}
\end{aligned}
$$

where $\gamma=\left(1-\beta^{2}\right)^{-1 / 2}$. Using these and the fact that in $S$ the orbit obeys $x^{2}+y^{2}=R^{2}$ one can describe the orbit in terms of $S^{\prime}$ variables as

$$
\frac{\left(x^{\prime}+\beta c t^{\prime}\right)^{2}}{R^{2}\left(1-\beta^{2}\right)}+\frac{y^{\prime 2}}{R^{2}}=1 .
$$

As can be inferred clearly from the above, in its own inertial co-ordinates the spaceship will describe the planetary orbit as an ellipse that moves to the left with speed $\beta c$ and with focal points located at $\pm \beta R \hat{y}$. However, the sun remains at the centre in clear disagreement with Kepler's first law.

The didactical merit of the example lies in its simplicity; it can be introduced right after discussing length contraction as a simple refutation of compatibility between special relativistic dynamics and laws of gravity in their Keplerian form.

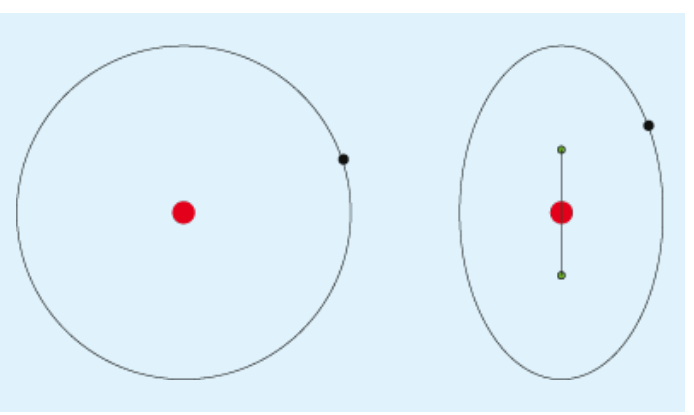

Figure 1. The circular orbit of the planet as seen by an inertial observer at rest with respect to the sun contrasted to the orbit as seen by an inertial observer moving with $\vec{v}=c \beta \hat{x}$. The moving observer describes the orbit as an ellipse with focal points at $\pm \beta R \hat{y}$. In both pictures notice that the sun remains at the centre and thus not generally at a focal point of the orbit.

We can elaborate; as far as equations of motion go special relativity is a generalization of Newtonian dynamics incorporating the invariance of the speed of light. The laws in their not manifestly covariant form are the same as in Newtonian dynamics

$$
\begin{gathered}
\frac{\mathrm{d} \overrightarrow{\boldsymbol{P}}}{\mathrm{d} t}=\overrightarrow{\boldsymbol{F}} \\
\frac{\mathrm{d} E}{\mathrm{~d} t}=\overrightarrow{\boldsymbol{F}} \cdot \vec{u}
\end{gathered}
$$

with the known relativistic definitions of momentum $\vec{P}$ and energy $E$. Now, as is the case with Newtonian dynamics, this does not tell us much about 
the nature [3] of $\vec{F}$; it can be obtained via input from physical phenomena, and there is no a priori reason why Kepler's first law for instance should not be applicable [4]. The example of this work if used as a homework assignment problem can also be extended by asking the direction of the force in $S^{\prime}$. The point of this extension would be to show that the force $\vec{F}^{\prime}$ cannot point towards a focal point because there are two of them and no conceivable way to choose a particular one strengthening the general argument. In fact, as expected, the Lorentz transformed force for the moving observer is always along the line joining the planet and the sun, although its magnitude may differ along the orbit.

We must be careful in assessing what notion this example will really provide the students with. Kepler's first law [5] is actually a statement about the orbits, not about the fundamental agent causing such orbits. The example that we have discussed emphasizes an important aspect about propositions of this form, which are not truly fundamental.

For instance, circular orbits are also present in electromagnetism; the same example can be discussed in view of Coulomb's law [6] and another relativistic observer will definitely see such an orbit as an ellipse where the point of attraction is not one of the foci [7]. However, the agent responsible for such an orbit, namely the Lorentz force, is fully compatible with special relativity; it has the same form for all relativistic inertial observers.

Furthermore Kepler's statement is in principle that the orbits are conic sections. If they are closed these can be either circles or ellipses. It is evident that because Lorentz transformations are linear, a closed conic curve will remain a closed conic curve. That is, the statement that the orbit is either a circle or an ellipse is relativistically invariant. Thus the true Achilles' heel of the law is the inclusion of the position of the sun or, for finite masses, of the position of the (Newtonian) centre of mass in the statement. Since the (Newtonian) centre of mass is not a relativistic concept we arrive at where exactly Kepler's first law fails to operate [8]. It is also amusing to remember in this context that Kepler's third law had to be adjusted in the Newtonian approach to account for the finite mass of the sun. Last but not least, an in-depth technical study of our simple example would require the full study of special relativistic two-body problem. This is a problem that is rather difficult and has been extensively studied with literature too wide to include here.

Does this example even slightly point towards general relativity? Certainly not. For instance, another observation is that since circular geodesics are not denied in Schwarzchild's [9] solution the very same observation we have made about the fact that the inertial observer $S^{\prime}$ describing the orbit as an ellipse is still present. As is well known, one of the best indicators of a need for a new theory of relativity is that gravity has to be a field theory consistent with the observation that there should be no immediate action at a distance and the principle of equivalence. The latter is incompatible with a framework dealing only with inertial observers. Nevertheless, in our example there seems to be a slight zest of a need to depart from special relativity if gravity is to be included in the picture, although we do not advocate that this should be emphasized in force in an undergraduate course. Furthermore, our example does not constitute a paradox in special relativity either. It simply means what it says; Kepler's first law is incompatible with special relativity.

As a conclusion we can say that the example we have discussed may be of use to undergraduate introductory courses in special relativity. From a simple observation it can point in various philosophical and technical directions; in this short note we have pointed out a few of these.

\section{References and notes}

[1] Pryce M H L 1948 Proc. Roy. Soc. Lon. 195 62-81

[2] Chryssomalakos C, Hernandez-Coronado $\mathrm{H}$ and Okon E 2009 J. Phys.: Conf. Ser. 174 012026

[3] There is, however, a strict condition on the Minkowski four-force $M^{\mu}$ containing $\vec{F}$. Since $P_{\mu} P^{\mu}$ is both an invariant and a constant of motion one can easily find that $M_{\mu} P^{\mu}=0$, which is most naturally achieved if $M^{\mu}=F^{\mu v} P_{v}$ where $F$ is an antisymmetric field strength. Such a constraint is absent in Newtonian dynamics and the most one can obtain for interactions between two bodies is that the force depends on the difference in their position vectors as a result of the observation that this difference is invariant under Galilean transformations.

[4] We will see that, a posteriori, propositions in 
the form of Kepler's first law can never hold between relativistic inertial frames.

[5] First called a law by Voltaire in Elements of Newton's Philosophy in 1738.

[6] For all practical purposes one can ignore the gravitational pull among the bodies next to the interaction between the charges. One can also cook up an instance where radiation due to acceleration is negligible.

[7] On the other hand, historically there was never a statement of Coulomb's law in the form of Kepler's statement.

[8] In fact even a relativistic generalization of the concept of centre of mass as, for instance, done by Pryce [1] yields non-commuting Poisson brackets of its co-ordinates and thus is not free of obstacles. See also [2] and the references therein.

[9] However, there is a lower bound below which circular orbits are unstable.

Tonguç Rador Boğaziçi University, Department of Physics, Bebek 34342, Istanbul, Turkey and Izmir Institute of Technology, Department of Physics, Urla 35430,Izmir,Turkey(e-mail tonguc.rador@ boun.edu.tr) 